\title{
ShaMAN: An Agent Meta-model for Computer Games
}

\author{
Steve Goschnick, Sandrine Balbo, and Liz Sonenberg \\ Interaction Design Group, DIS, University of Melbourne, 3010, Australia \\ \{stevenbg, sandrine, 1.sonenberg\} @unimelb. edu . au
}

\begin{abstract}
In this paper, we detail recent research on agent meta-models. In particular, we introduce a new agent meta-model called ShaMAN, created with a specific focus on computer game development using agent systems. ShaMAN was derived by applying the concept of Normalisation from Information Analysis, against a superset of agent meta-model concepts from the meta-models investigated. A number of features are identified, including human-agent locales and socialworlds, that might be usefully added to a generic AO meta-model.
\end{abstract}

Keywords: Agent-oriented, Agent Architecture, Multi-Agent Systems, Metamodel, Agent Meta-models, Agents in Computer Games, HCI.

\section{Introduction}

Agent-oriented (AO) architectures and methodologies are the main interest area of the research outlined here, with a focus on the application domain of computer games. While we are specifically interested in extending current $\mathrm{AO}$ concepts to further facilitate game specification and development, a consequence of this study identifies possible generic features to add to an AO meta-model.

\subsection{Motivation}

Computer games invariably have a graphic user interface (GUI) whether they are on PCs, dedicated game consoles or mobile phones. Additionally, many games are multiuser over either a proprietary network or the Internet, and as such, some data is often shared between multiple users. Neither graphic interfaces nor their associated event models, nor distributed data are well considered in the current AO architectures and frameworks, but computer games make heavy use of all three.

There is a precedent early on in the Object-oriented (OO) paradigm for an underappreciation of these same facets of application programs, which ought to be instructive for the newer AO paradigm. At about the time that mainstream developers moved to OO languages, in particular C++ (early 1990's), GUI interfaces became the default in mainstream operating systems (OS). GUI and mouse/pointer interfaces made it necessary for application programmers to handle non-sequential event-handling, a significant change in programming practice from sequential processing in most character-based applications. Prior to more modern OO languages such as Java, both the GUI and event-handling was not a part of the language proper, e.g. C++. For 
exam-ple, on the Unix OS events were handled via X-Windows and Motif class libraries. Thus, the application programmer in the early 1990s moved to an OO paradigm in language constructs, but their dealings with the GUI and event-driven programming, initially happened outside of the OO paradigm. So, an event-driven paradigm of pro-gramming happened concurrently by necessity, but it initially went unheralded in the shadow of the OO language paradigm.

\subsection{A Gap in Agent Architectures}

From the start AO has been socially-oriented such that inter-agent communication - a form of event - is typically allowed for with an Agent Communication Language (ACL). However, the AO paradigm has followed the initial OO programming languages, in not doing anything within the architecture or the constructs of the languages themselves, with regard to the GUI interface or non-agent event-handling.
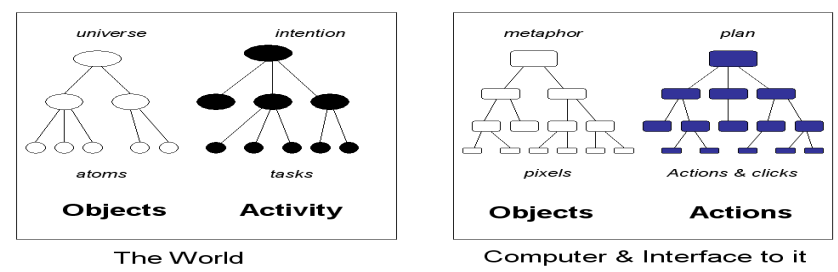

Fig. 1. Real world and the Object Action Interface Model

Interaction events and GUI interface objects are at the core of all mainstream computing platforms today, whether it be workstation, desktop, laptop, PDA or mobile phone. Figure 1 is an adaption of Shneiderman's Object Action Interface Model [20], showing a high-level representation of the physical world and what is done on a computer to supplement it, when a user interacts with an application program, via a computer screen and input mechanisms (e.g. mouse and keyboard).

The gap addressed by our research, is to achieve an AO architecture that engages with the user at the level of a GUI metaphor rendered down to the pixel level (lefthand side of right box in figure 1), with events down to the keyboard and mouse-click level, (right-hand side of figure 1). Our architecture is expressed as a meta-model.

\subsection{Meta-models}

Much of the research discussed here is centred around meta-models expressed in UML class diagram notation. Meta-models expressed in UML as such are now commonly used in both $\mathrm{AO}[1,12,13]$ and $\mathrm{OO}[17]$ research and development domains: to represent state-holding entities; to communicate base ideas; and as a useful means to compare different agent systems or architectures $[6,12]$.

\subsubsection{Agent Concepts}

Given that there is currently no universally accepted single meta-model for AO systems, when we first looked to agent concepts and architectures with computer games 
in mind, we examined the meta-models of several agent architectures and methodologies - AAII [16], GAIA [22,23], Tropos [1,11], TAO/MAS-ML [5], ROADMAP $[13,14]$, ShadowBoard $[8,9]$ - to explore the commonalities and differences between them. In addition, given our identification of a gap in the AO paradigm at the input device event level, we studied several well-known meta-models from the Task Modelling field, with its roots in the interaction between human users and computational devices, covered elsewhere [10].

\subsubsection{Normalisation}

A technique from Information Analysis (IA) used to improve ER models [2] that did not crossover into the later OO paradigm is the concept of Data Normalisation [15]. In this process derived from relational mathematics by Codd [3], the ER model is put into normal form. The model resulting from normalising a preliminary model, is considered to be in a state ideal for future change, and one that causes the least anomalies to operations upon the state held in the current entities. It is usually applied in IA to a model as a quality control procedure, however, Normalisation can also be used as a bottom-up design technique enabling the analyst to methodically deduce a wellformed model from a set of relevant concepts. In this research we applied it to a superset of the agent concepts found in agent and task meta-models, and arrived at a normalised agent meta-model named ShaMAN. From the perspective of a multi-agent system at runtime, a normalised meta-model is best for insertion, update and deletion of state information as it is happening in real-time.

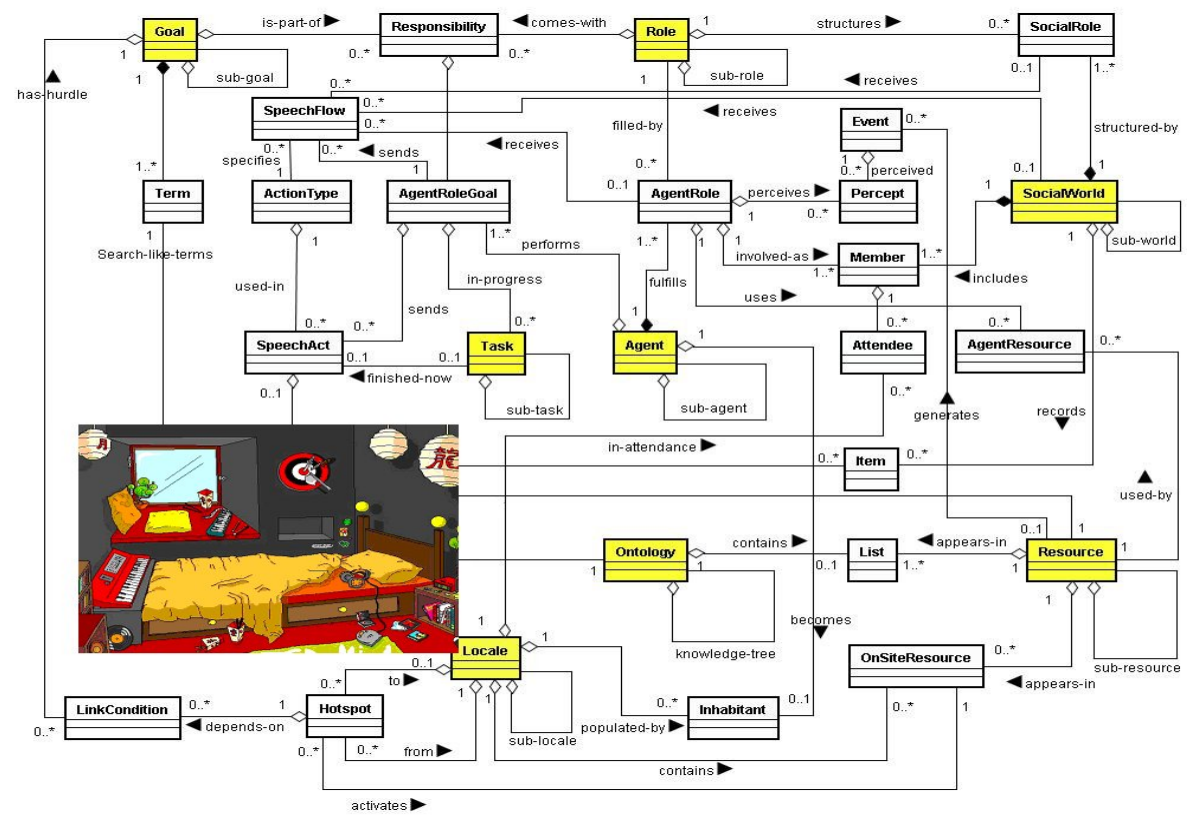

Fig. 2. The ShaMAN Agent Meta-model (with insert of a concrete game Locale) 


\subsubsection{Overview}

In Section 2 we introduce the ShaMAN agent meta-model. To explain some of the entities in it, we present two groupings of the entities from the meta-model in detail, and then describe the flexibility it brings to building applications. In Section 3 we compare the concepts from the ShaMAN meta-model with those other agent meta-models investigated. In Section 4 we conclude and look to future work related to ShaMAN.

\section{The ShaMAN Meta-model}

We arrived at the ShaMAN meta-model depicted in figure 2 by taking concepts from a number of existing AO meta-models and a number of Task Analysis meta-models [10] - analysed them for similarities and differences, added some extra requirements from the games application genre, and then normalised the resultant set of entities. The following sections describe some aspects of the ShaMAN meta-model in more detail.

\subsection{Locales for Computer Games}

Computer games invariably interact with the player through the usage of a humanmachine interface, for example a screen of one size or another. The Locale sub-section of ShaMAN lets us model the visual metaphors and the screen interaction between player/user and screen characters of a game, right in the AO model itself, rather than leaving it to some other paradigm such as OO. While some agent meta-models do have constructs for the agent environment, none of those investigated specifically model the computer screen as the primary representation of that environment.

In ShaMAN, this screen representation of an agent's environment is called a Locale - in homage to Fitzpatrick's [7] definition of a Locale as a generalised abstract representation of where members of a Social World [21] inhabit and interact. Figure 3 represents the sub-section of the ShaMAN meta-model that represents Locales within games.

A Locale entity may have sub-locales within hierarchies of Locales. Locale is a generic concept representing some spatial construct presentable on the screen, e.g. room, outdoor area, sections of a board-game - suitably broad enough for novel game interfaces.

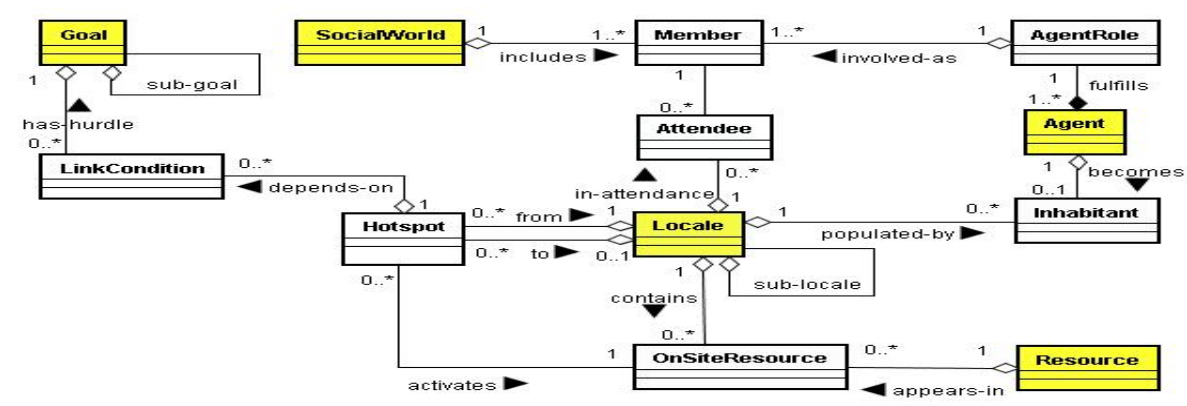

Fig. 3. The Locale sub-section of the meta-model 
The insert in figure 2 is a concrete example of a Locale. It depicts the bedroom of a player's character within a game, which is represented as a Locale in ShaMAN. The HotSpot entity represents any area on the screen that is interactive, in the sense that whenever the user either clicks or passes over that area on the screen (or has the focus, from a keystroke point-of-view), certain interaction between the user and the game may take place. Whether the game presents a $2 \mathrm{D}$ or $3 \mathrm{D}$ scene, or an abstraction, the interaction with a standard display is 2D and involves area. The HotSpot entity has two relationships with Locale, one named to and the other named from - enabling navigation between Locales.

A HotSpot may also link to an OnSiteResource entity. These are Resources that live in the Resource entity (which may involve a hierarchy of Resources). Resources are typically programmed entities that are not Agent-oriented. E.g. clicking on the digital clock on the bedside table opens a window that displays a fully-functioning clock object, which is a Resource. OnSiteResource is an associate entity - a representation that allows the same Resource to be used in multiple Locales, e.g. a clock in many rooms drawing upon the same programmed code. Resource may also represent real objects in the real world, such as in a robot or a sensor application based upon the ShaMAN meta-model.

A HotSpot may also have a relationship with the entity LinkCondition, which in turn links to a Goal via a relationship called has-hurdle. This allows the game developer to enforce conditions to be met. Locale is also linked to the entities Attendee and Inhabitant. Attendee is an associative entity that records all occupants in a particular Locale over time, retaining a record of when agents (or human avatars) entered and left a Locale. It is linked to the agent's Role during that occupation via AgentRole, and also to the SocialWorld they were engaged in when they did so. This history aspect of the Attendee is usefull in providing and/or recording a back-story for any particular agentoriented game character - a necessary aspect of realistic game creation.

\subsection{The Goals, Roles, Responsibilities and Tasks of Agents}

Computer games often have the need for intelligent, intentional, proactive and autonomous game characters that interact both with the human players and with other char-acters in a game. These properties are the harbingers of AO systems, and the sub-group of entities from ShaMAN meta-model in left and centre of figure 4,

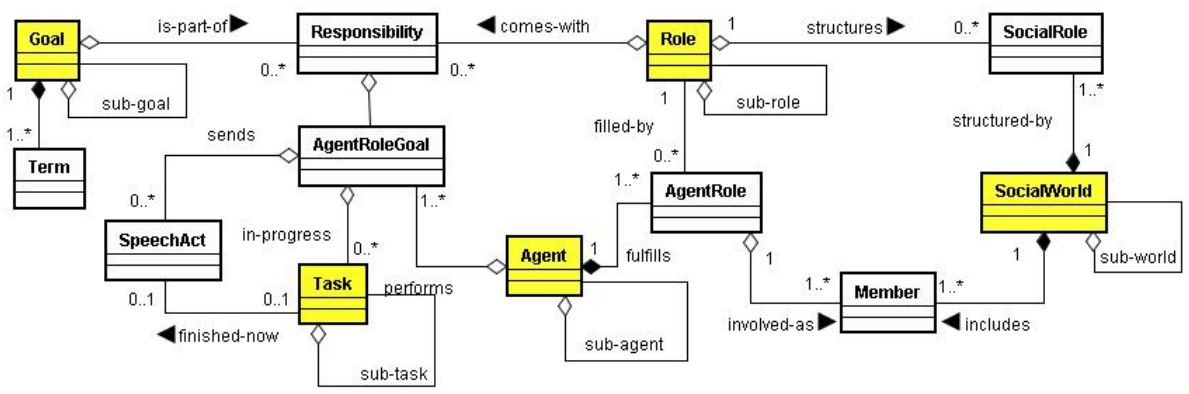

Fig. 4. Goals, Roles and Tasks in ShaMAN 
represent the entities that appear most frequently (but not consistently) in one form or another, in many of the agent meta-models that we examined.

Figure 4 shows five entities in this sub-model of ShaMAN that have hierarchies of sub-elements of the same type, namely: Goal, Role, Agent, Task and SocialWorld. The associate entity between Goal and Role called Responsibility represents the responsibilities of a particular Role. A given Responsibility instance is fulfilled via an

Table 1. ShaMAN meta-model comparison with other agent architectures and meta-models

\begin{tabular}{|c|c|c|c|c|c|c|c|}
\hline ShaMAN & KGR /BDI & GAIA V1 & GAIA V2 & RoadMap & Tropos & $\begin{array}{l}\text { MAS- } \\
\text { ML }\end{array}$ & DigitalFriend \\
\hline AgentRole & & Role & Role & (pointers) & & Play & AgentRole \\
\hline Percept & & & & & & & Percept \\
\hline Event & & & & & & Event & Event \\
\hline $\begin{array}{l}\text { SocialWorld } \\
\text { (tree) }\end{array}$ & $\begin{array}{l}\text { Acquaint- } \\
\text { ance }\end{array}$ & System & $\begin{array}{l}\text { Organisation, } \\
\text { Pattern }\end{array}$ & & $\begin{array}{l}\text { Actor/ } \\
\text { Social } \\
\text { Agent }\end{array}$ & $\begin{array}{l}\text { Organisa- } \\
\text { tion }\end{array}$ & \\
\hline SocialRole & & System & & & $\begin{array}{l}\text { Actor/ } \\
\text { Position }\end{array}$ & $\begin{array}{l}\text { Owner- } \\
\text { ship }\end{array}$ & \\
\hline \multicolumn{8}{|l|}{ Member } \\
\hline \multicolumn{8}{|l|}{ Item } \\
\hline $\begin{array}{l}\text { AgentRole- } \\
\text { Goal }\end{array}$ & $\begin{array}{l}\text { Capability, } \\
\text { Service }\end{array}$ & $\begin{array}{l}\text { Services, } \\
\text { Activity }\end{array}$ & $\begin{array}{l}\text { Service, } \\
\text { Activity }\end{array}$ & Services & $\begin{array}{l}\text { Depend- } \\
\text { ency }\end{array}$ & Actions & AgentRoleGoal \\
\hline Task (tree) & & Activity & Activity & & Plan & & Task \\
\hline SpeechAct & Interaction & Activity & & & & Message & SpeechAct \\
\hline ActionType & $\begin{array}{l}\text { Action, } \\
\text { performa- } \\
\text { tive }\end{array}$ & & & & & & Action \\
\hline SpeechFlow & $\begin{array}{l}\text { Acquaint- } \\
\text { ance, } \\
\text { Permis- } \\
\text { sions, } \\
\text { Protocols }\end{array}$ & $\begin{array}{l}\text { Protocol, } \\
\text { Acquaintance } \\
\text { model }^{5}\end{array}$ & Protocol & & & Protocol & MessageFlow \\
\hline $\begin{array}{l}\text { Resource } \\
\text { (tree) }\end{array}$ & Resource & Resource & Resource & $\begin{array}{l}\text { Protocol } \\
\text { (tree) }\end{array}$ & Resource & Object & Resource \\
\hline $\begin{array}{l}\text { Agent- } \\
\text { Resource }\end{array}$ & Service & Permissions & Permissions & & $\begin{array}{l}\text { Depend- } \\
\text { ency }\end{array}$ & & AgentResource \\
\hline $\begin{array}{c}\text { Ontology } \\
\text { (tree) }\end{array}$ & & & & $\begin{array}{l}\text { Knowledge- } \\
\text { Component }\end{array}$ & & & Ontology (tree) \\
\hline List & Knowledge & & & & & & ResourcList \\
\hline Locale (tree) & & & Environment & $\begin{array}{l}\text { Environment- } \\
\text { Zone }\end{array}$ & & $\begin{array}{l}\text { Environ- } \\
\text { ment }\end{array}$ & \\
\hline \multicolumn{8}{|l|}{ Attendee } \\
\hline Inhabitant & & & & & & Inhabit & \\
\hline \multicolumn{8}{|l|}{$\begin{array}{l}\text { OnSite } \\
\text { Resource }\end{array}$} \\
\hline \multicolumn{8}{|l|}{ HotSpot } \\
\hline \multicolumn{8}{|l|}{$\begin{array}{l}\text { Link- } \\
\text { Condition }\end{array}$} \\
\hline$R, A, D, I, T, R t$ & $\mathrm{~A}, \mathrm{D}, \mathrm{I}$ & $\mathrm{A}, \mathrm{D}$ & A,D & $\mathrm{R}, \mathrm{A}, \mathrm{D}, \mathrm{Rt}$ & $\mathrm{R}, \mathrm{A}, \mathrm{D}, \mathrm{I}$ & R,A,D & $\mathrm{R}, \mathrm{A}, \mathrm{D}, \mathrm{I}, \mathrm{Rt}$ \\
\hline
\end{tabular}

Note 1. R,A,D,I,T,Rt lifecycle phases: Requirements, Analysis, Design, Implementation, Testing, Run-time. Note 2. The DigitalFriend V1 tool [9] is an implementation of the ShadowBoard agent architecture [8].

Note 3. AO models for Prometheus [18] and GoalNet [19] were in the study but not here, for space rea-sons. 
instance in the AgentRoleGoal entity, by being enacted or performed by an Agent that takes on that Role. An Agent may have many Roles via AgentRole.

Goals will often have sub-goals in a hierarchy of goals to be achieved. One such sub-goal will be associated with a matching sub-role, and an agent will be assigned via an instance of the AgentRole entity. During execution of a ShaMAN application, sub-agents can be called upon in a downward direction via the need to achieve the sub-goals of parent goals, which is termed goal-driven execution. Or, they can be called upon from below, where a SpeechAct has been sent from further down the subagent chain, and the upper level goal has to be solved or rerun, termed data-driven execution. Data-driven execution often eventuates when a sub-agent retrieves new information from an external service such as a Web service, or from another agent across agent hierarchies or across Social Worlds.

\subsection{Social Worlds in ShaMAN}

Individual Agents can be members of one or more SocialWorlds. Their membership begins with an instance in the Member entity. Agents are related to the Member entity via the AgentRole entity. SocialWorld's have a number of SocialRoles, such as 'Captain' or 'Treasurer', which is useful in the design phase before specific agents are instantiated.

\section{A Comparison of Agent Meta-models}

Our motivation for collecting and comparing agent meta-models was for their agent concepts, as the primary input into a normalisation process, to arrive at a well-formed agent meta-model. Hence our initial interest in the comparison was analytic only.

Table 1 is a comparative format representing a sub-set of agent concepts that we used as input into the meta-model normalisation process in deriving the ShaMAN entities (the first table column). All models have some entity similar to the ShaMAN's Goal, Role, Responsibility and Agent, so these have been excluded from the table in this paper. Even so, a particular comparison (e.g. ShaMAN's Goal(tree) and Tropos's Soft Goal/Hard Goal) only approximately equates the concepts. Sometimes a comparison is close in meaning, other times it is close in name but distant in meaning, and sometimes there is wide variance in both name and the semantics. In the full study we did examine each twin comparison of concept in detail, but it cannot be presented in this paper for space reasons.

What is more useful in this paper is to highlight where ShaMAN has entities that have little or no comparison across the other agent meta-models examined. The darker shaded cells in the table shows that entities around Locale are unique to ShaMAN. Similarly, the lighter shaded cells show several of the entities related to SocialWorld are unique to ShaMAN. These entities were discussed explicitly above in the discussion of figures 3 and 4 .

\section{Conclusions and the Future}

Agent-oriented architectures and frameworks lend themselves well to Human-Centred Software Engineering, given that several of them are derived from branches of 
psychology and mentalistic notions (e.g. BDI - from Folk Psychology; ShadowBoard from Analytical Psychology). We set out to extend current AO concepts to further facilitate game specification and development. While the entities unique to ShaMAN were introduced specifically for that purpose, most of them have a more generic usage, particularly for intelligent applications, with multiple users, many agents and rich user interfaces. It has not been our intent to develop a generic agent meta-model, however others are endeavouring to define an all-inclusive agent meta-model: Hahn et al [12] demonstrate the usefulness of the MDA (model driven architecture, see OMG [17]) approach to software development with AO tools. Fischer et al [6] propose that a unified agent meta-model is a worthy goal and could provide interoperability between many of the current disparate agent meta-models, methodologies and technology platforms. Theirs is a work-in-progress that we intend to align ShaMAN development with, as much as possible.

\section{References}

1. Bresciani, P., Perini, A., Giorgini, P., Guinchiglia, F., Mylopoulos, J.: Tropos: An AgentOriented Software Development Methodology. Autonomous Agents and Multi-Agent Systems, 203-236 (2004)

2. Chen, P.: The Entity-Relationship Model - Toward a Unified View of Data. ACM Transactions on Database Systems 1, 9-36 (1976)

3. Codd, E.F.: A relational model of data for large shared data banks. Communications of the ACM 13, 377-387 (1970)

4. Cossentino, M.: Different perspectives in designing multi-agent systems. In: AgeS 2002, workshop at NodE 2002, Erfurt, Germany (2002)

5. Da Silva, V.T., De Lucena, C.J.P.: From a Conceptual Framework for Agents and Ob-jects to a Multi-Agent System Modeling Language. Autonomous Agents and Multi-Agent Systems 9, 145-189 (2004)

6. Fischer, K.: Agent-oriented software engineering: a model-driven approach. International Journal of Agent-Oriented Software Engineering 1(3/4), 334-369 (2007)

7. Fitzpatrick, G.: The Locales Framework: Understanding and Designing for Wicked Problems. Kluwer Academic Publications, London (2003)

8. Goschnick, S.B.: ShadowBoard: an Agent Architecture for enabling a sophisticated Digital Self. Thesis, Dept. of Computer Science, University of Melbourne, Australia (2001)

9. Goschnick, S.B.: The DigitalFriend: the First End-User Oriented Multi-Agent System. In: OSDC 2006, the third Open Source Developers' Conference, Melbourne, Australia, December 5-8 (2006)

10. Goschnick, S., Balbo, S., Sonenberg, L.: From Task to Agent-Oriented Meta-models, and Back Again. In: Tamodia 2008, Pisa, Italy (2008)

11. Guinchiglia, F., Mylopoulos, J., Perini, A.: The Tropos Software Development Methodology: Processes, Models and Diagrams. In: AAMAS 2002. ACM, New York (2002)

12. Hahn, C., Madrigal-Mora, C., Fischer, K., Elvesaeter, B., Berre, A., Zinnikus, I.: Metamodels, Models, and Model Transformations: Towards Interoperable Agents. In: Fischer, K., Timm, I.J., André, E., Zhong, N. (eds.) MATES 2006. LNCS (LNAI), vol. 4196, pp. 123-134. Springer, Heidelberg (2006)

13. Juan, T., Sterling, L.: The ROADMAP Meta-model for Intelligent Adaptive Multi-Agent Systems in Open Environments. In: 4th International Workshop on Agent Oriented Software Engineering, Melbourne (2003) 
14. Juan, T., Pearce, A., Sterling, L.: ROADMAP: Extending the Gaia Methodology for Complex Open Systems. In: Autonomous Agents and Multi-Agent Systems AAMAS 2002 (2002)

15. Kent, W.: A Simple Guide to Five Normal Forms in Relational Database Theory. Communications of the ACM 26(2), 120-125 (1983)

16. Kinny, D., Georgeff, M., Rao, A.: A Methodology and Modelling Technique for Systems of BDI Agents. In: Van de Velde, W., Perram, J.W. (eds.) The Seventh European Workshop on Modelling Autonomous Agents in a Multi-Agent World. Springer, Berlin (1996)

17. OMG: MDA Guide Version 1.0.1 (2003), http//www. omg.org/docs / omg/03-06-01.pdf

18. Padgham, L., Winikoff, M.: Prometheus: A Methodology for Developing Intelligent Agents. In: AOSE Workshop, AAMAS-20, Bologna, Italy (2002)

19. Shen, Z., Li, D., Miao, C., Gay, R.: Goal-oriented Methodology for Agent System Development. In: International Conference on Intelligent Agent Technology, IAT 2005 (2005)

20. Shneiderman, B.: Designing the User Interface, Strategies for Effective Human-Computer Interaction, 3rd edn. Addison-Wesley, USA (1997)

21. Strauss, A.: A Social World Perspective. Studies in Symbolic Interaction 1, 119-128 (1978)

22. Wooldridge, M., Jennings, N.R., Kinny, D.: The Gaia Methodology for Agent-Oriented Analysis and Design. Autonomous Agents and Multi-Agent Systems 3, 285-312 (2000)

23. Zambonelli, F., Jennings, N.R., Wooldridge, M.J.: Developing Multiagent Systems: The Gaia Methodology. ACM Transactions on Software Engineering and Methodology 12, 417-470 (2003) 